\title{
A general, norbornyl based approach to anti-Bredt alkenes via sequential RCM-fragmentation strategy
}

\author{
Goverdhan Mehta* and R. Senthil Kumaran \\ Department of Organic Chemistry, Indian Institute of Science, Bangalore 560 012, India
}

Received (in Cambridge, UK) 15th April 2002, Accepted 16th May 2002

First published as an Advance Article on the web 6th June 2002

A general protocol for the synthesis of bicyclo[n.3.1]frameworks with bridgehead double bond (anti-Bredt alkenes), from a common, readily available norbornyl precursor, involving sequential ring closure metathesis (RCM) and Wharton fragmentation is outlined.

For several decades now, an assortment of bridgehead olefins (anti-Bredt alkenes) ${ }^{1}$ of which bicyclo[3.3.1]non-1(2)-ene $\mathbf{1}^{2 a, b}$ and bicyclo[3.2.2]non-1(7)-ene $\mathbf{2}^{2 c}$ are prototypes, have been challenging targets of synthesis as these molecules provide insights into the strain induced distortions about the double bond and its manifestation in chemical reactivity. ${ }^{1,2}$ More recently, many prominent natural products like taxol ${ }^{\circledR} \mathbf{3}$ and CP263,1144 , notable for structural complexity and biological
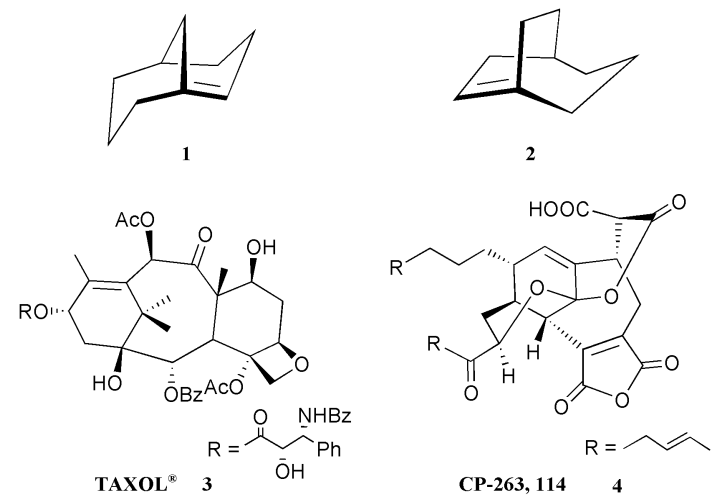

activity, have been found to incorporate bridgehead double bonds in the form of a bicyclo[5.3.1] undec-1(10)-ene and a bicyclo[4.3.1]dec-1(9)-ene core, respectively, embedded within their structure. This has further stimulated widespread interest in the synthesis of bicyclo[n.3.1]frameworks embodying a bridgehead double bond. ${ }^{3-6}$ Several strategies have been recently reported in the literature that provide access to bridgehead alkenes based on bicyclo[5.3.1]- and bicyclo[4.3.1] frameworks, in the context of the synthesis of taxoids ${ }^{3}$ and $\mathrm{CP}$ molecules ${ }^{4}$ but general methodologies towards antiBredt alkenes are rather limited. $5 a, c, 6 c \mathrm{We}$ have been enticed by this area and report a new, general approach to bicyclo[n.3.1]framework based bridgehead olefins (anti-Bredt alkenes) which emanates from a norbornyl platform $\mathbf{5}$ and involves in sequential ring closure metathesis $(\mathrm{RCM}, \mathbf{5} \rightarrow \mathbf{6})$ and Wharton fragmentation $(\mathbf{7} \rightarrow \mathbf{8})$ as the pivotal steps as shown in Scheme 1.

The key element of the approach delineated in Scheme 1 is the assembly of the norbornyl precursor $\mathbf{5}$ in which the two alkene arms at $\mathrm{C} 2$ and $\mathrm{C} 7$ are projected on the exo- and synface, respectively, in order to facilitate the RCM to the bridged tricyclic system $\mathbf{6}$. When the unprotected hydroxy group in $\mathbf{6}$ is activated as in $\mathbf{7}$, a facile fragmentation can be expected to deliver bridgehead alkene 8 with high level of functionalization. Successful execution of this scheme forms the subject matter of this communication.

Our synthetic approach emanated from the keto-acetate 9, a readily available 2,7-disubstituted norbornyl derivative. ${ }^{7}$ Addi-

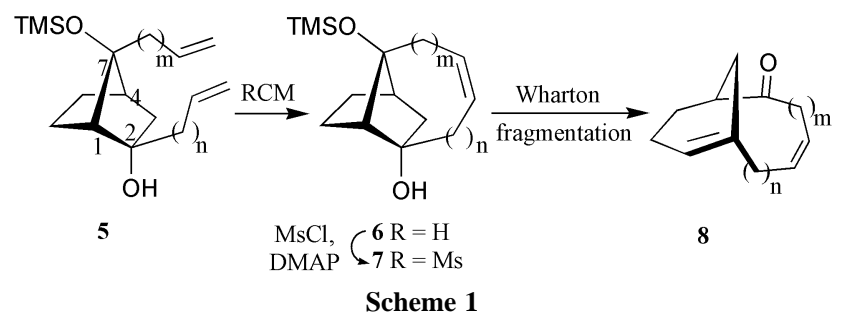

tion of vinyl magnesium bromide to 9 did not exhibit significant facial discrimination ${ }^{8}$ and base hydrolysis furnished anti- and syn- addition products $\mathbf{1 0}$ and $\mathbf{1 1}(45: 55)$, Scheme $2.9^{9}$ The secondary hydroxy group in syn-11 was oxidised and the tertiary hydroxy group was protected to furnish 12. Further vinylation of the $\mathrm{C} 2$ carbonyl in $\mathbf{1 2}$ led to a readily separable mixture of endo- and exo-addition products 13 and $\mathbf{1 4}(58: 42) .{ }^{9}$ The requisite exo-, syn-divinyl compound 14 on exposure to Grubbs' catalyst [benzylidene-bis(tricyclohexylphosphine)-dichlororuthenium] ${ }^{10}$ underwent smooth $\mathrm{RCM}^{11}$ to furnish the tricyclic olefin $1^{9}$ bearing the brexane framework, Scheme 3. Catalytic hydrogenation of $\mathbf{1 5}$ to $\mathbf{1 6}$ and mesylation furnished the tricyclic endo-mesylate 17.9 TMS- deprotection to $\mathbf{1 8}$ and exposure to base resulted in a smooth fragmentation, as contemplated, to deliver the anti-Bredt bicyclo[3.3.1]non1(8)-en-4-one $1^{9}$ in good yield, Scheme 3.

In the backdrop of the successful acquisition of 19, we looked for the generalization of this protocol. Accordingly, the ketoacetate 9 was subjected to allylation under Barbier conditions employing different metals to fine tune face-selectivity and obtain better access to the desired syn isomer 20, Scheme 4. Allylation in the presence of zinc proved to be the best option with 68:32 ratio of $\mathbf{2 0}$ and $\mathbf{2 1} .^{9}$ The syn isomer $\mathbf{2 0}$ was further elaborated to the TMS-protected ketone 22 through a series of straightforward transformations, Scheme 5. Further allylation of $\mathbf{2 2}$ proceeded cleanly but the steric factors dominated the addition to give 23 and $24(3: 1)^{9}$ in which the required diastereomer formed through exo-addition was the minor product, Scheme 5. Nonetheless, $\mathbf{2 4}$ underwent smooth RCM in the presence of the Grubbs' catalyst ${ }^{10}$ to furnish the tricycle $\mathbf{2 5},{ }^{9}$

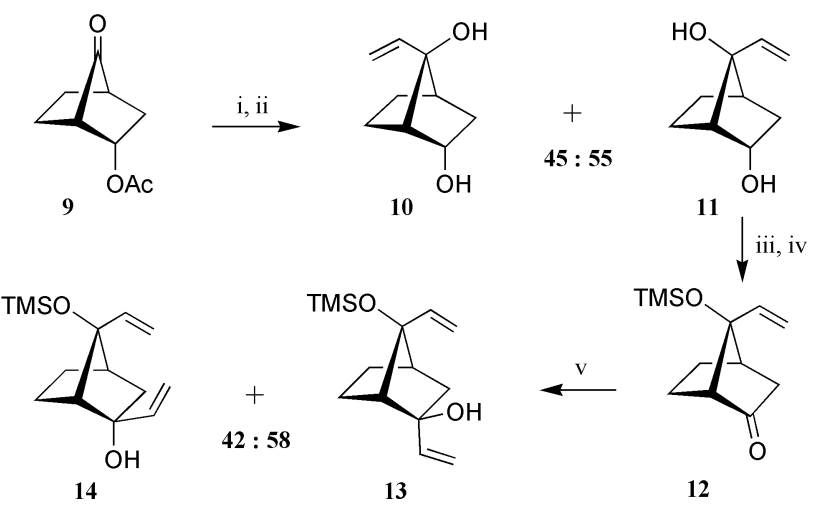

Scheme 2 Reagents and conditions: (i) $\mathrm{CH}_{2}=\mathrm{CHMgBr}$, THF, $0{ }^{\circ} \mathrm{C}, 75 \%$; (ii) $\mathrm{K}_{2} \mathrm{CO}_{3}, \mathrm{MeOH}, \mathrm{rt}, 80 \%$; (iii) TPAP, DCM, NMMO, 90\%; (iv) TMSCl, $\mathrm{Et}_{3} \mathrm{~N}, \mathrm{DCM}, 90 \%$; (v) $\mathrm{CH}_{2}=\mathrm{CHMgBr}$, THF, $80 \%$. 


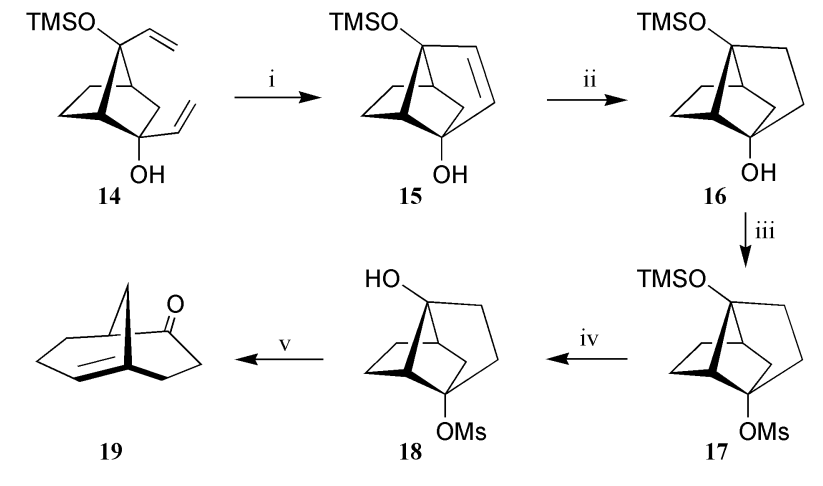

Scheme 3 Reagents and conditions: Grubb's catalyst $(30 \mathrm{~mol} \%), \mathrm{C}_{6} \mathrm{H}_{6}$, reflux, 83\%; (ii) $10 \% \mathrm{Pd} / \mathrm{C}, \mathrm{H}_{2}$, EtOAc, 95\%; (iii) $\mathrm{MsCl}, \mathrm{Et}_{3} \mathrm{~N}, \mathrm{DMAP}$ DCM, $0{ }^{\circ} \mathrm{C}, 85 \%$; (iv) TBAF, THF, rt, $80 \%$; (v) NaH, THF, $0{ }^{\circ} \mathrm{C}$ to rt, $87 \%$.
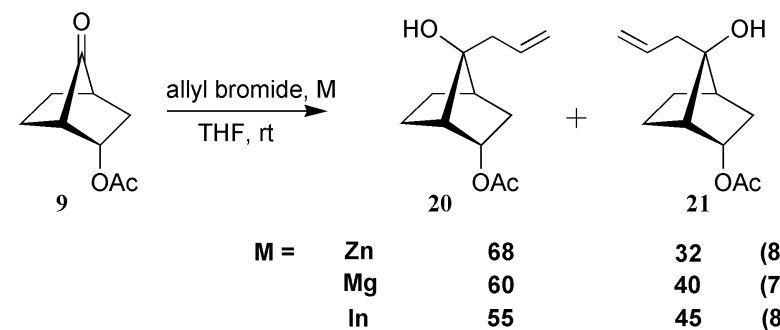

$\begin{array}{ll}\mathrm{Zn} & 68 \\ \mathrm{Mg} & 60 \\ \mathrm{In} & 55\end{array}$

40

$(80 \%)$

Scheme 4

Scheme 5. Under the conditions of mesylation, 25 underwent ready fragmentation to yield bicyclo[5.3.1]undeca1(10),3-dien-6-one 26, Scheme 5. The skeleton of the bridgehead alkene $\mathbf{2 6}^{9}$ is reminiscent of the bicyclic $\mathrm{AB}$ ring core of taxoids. $^{3}$

Our last example is of access to the bicyclo[4.3.1]dec2(9)-ene framework present in the $\mathrm{CP}$ molecules ${ }^{4}$ and originates from the syn-allyl ketone $\mathbf{2 2} .{ }^{9}$ Vinylation of $\mathbf{2 2}$ was again dominated by steric considerations and furnished endo- and exo- addition products 27 and 28 (73:27). ${ }^{9}$ The exo, syn-28 readily underwent RCM in the presence of Grubbs' catalyst ${ }^{10}$ to furnish the tricyclic olefin 29,9 Scheme 6. As the direct fragmentation on 29 was unsuccessful, perhaps due to strain factors, the double bond in it was reduced to furnish $\mathbf{3 0}$. Exposure of $\mathbf{3 0}$ to methanesulfonyl chloride in the presence of

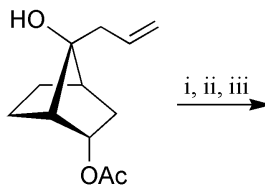

20

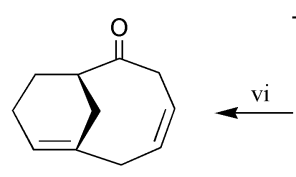

26<smiles>C=CCC1(OC)C2CCCC1C(=O)C2</smiles>

22

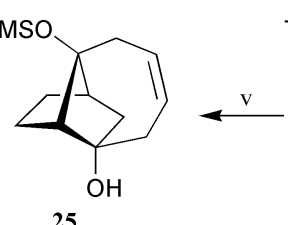

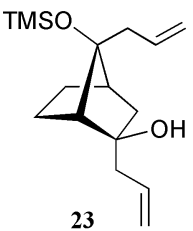

十 78:22

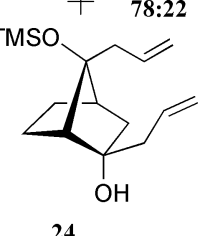

24
Scheme 5 Reactions and conditions: (i) $\mathrm{K}_{2} \mathrm{CO}_{3}, \mathrm{MeOH}$, rt,78\%; (ii) $\mathrm{PCC}$ DCM, rt, 80\%; (iii) TMSCl, Et ${ }_{3} \mathrm{~N}, \mathrm{DCM}, \mathrm{rt}, 91 \%$; (iv) allyl phenyl ether, $\mathrm{Li}$, $\mathrm{Et}_{2} \mathrm{O}-\mathrm{THF}, 0{ }^{\circ} \mathrm{C}$ to rt, 76\%; (v) Grubb's catalyst (30 mol\%), $\mathrm{C}_{6} \mathrm{H}_{6}$, reflux, $90 \%$; (vi) $\mathrm{MsCl}, \mathrm{Et}_{3} \mathrm{~N}$, DMAP, DCM, $0{ }^{\circ} \mathrm{C}, 75 \%$.

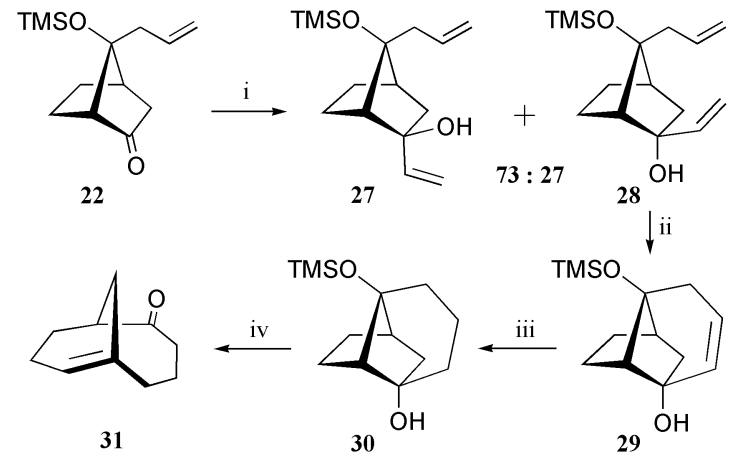

Scheme 6 Reactions and conditions: (i) $\mathrm{CH}_{2}=\mathrm{CHMgBr}$, THF, rt, 30-35\% (ii) Grubb's catalyst $(30 \mathrm{~mol} \%), \mathrm{C}_{6} \mathrm{H}_{6}$, reflux, 93\%; (iii) $10 \% \mathrm{Pd} / \mathrm{C}, \mathrm{H}_{2}$ EtOAc, $95 \%$; (iv) $\mathrm{MsCl}, \mathrm{Et}_{3} \mathrm{~N}$, DMAP, DCM, $0{ }^{\circ} \mathrm{C}, 85 \%$

base resulted in fragmentation to furnish bicyclo[4.3.1]dec2(9)-en-5-one 316c,9 in good yield, Scheme 6.

In summary, we have described a new, general approach to bicyclo[n.3.1]alk-1-enes with bridgehead double bond and additional functionalization on the framework from a single, readily available norbornyl precursor. We have employed ring closure metathesis (RCM) reaction on suitably crafted 2,7-disubstituted norbornyl derivatives, with syn disposed alkene arms, to generate the tricyclo $\left[n \cdot 3 \cdot 0 \cdot 0^{3, n+3}\right]$ system which is tailored to orchestrate a Wharton fragmentation to deliver the anti-Bredt alkenes. The limiting factor at the moment to this otherwise promising sequence (Scheme 1) is the control of stereochemistry of the two alkene arms on the norbornyl framework and this issue is the object of our current attention.

We thank JNCASR for the financial support. R. S. K. thanks CSIR for the award of a research fellowship.

\section{Notes and references}

1 Reviews: (a) K. J. Shea, Tetrahedron, 1980, 36, 1683; (b) P. M. Warner, Chem. Rev., 1989, 89, 1067.

2 (a) J. A. Marshall and H. Faubl, J. Am. Chem. Soc., 1967, 89, 5965; (b) J. R. Wiseman, J. Am. Chem. Soc., 1967, 89, 5966; (c) J. R. Wiseman and J. A. Chong, J. Am. Chem. Soc., 1969, 91, 7775.

3 For reviews on the synthesis of taxoids, see: (a) A. N. Boa, P. R. Jenkins and N. J. Lawrence, Contemp. Org. Synth., 1994, 47; (b) K. C. Nicolaou, W- M. Dai and R. K. Guy, Angew. Chem., Int. Ed. Engl., 1994, 33, 15; (c) G. Mehta and V. Singh, Chem. Rev., 1999, 99, 881

4 For a recent review on the synthesis of CP molecules, see: J. T. Starr and E. M. Carriera, Angew. Chem., Int. Ed., 2000, 39, 1415.

5 Selected examples of recent approaches to the bridgehead alkenes, see: (a) S. L. Gwaltney; S. T. Sakata and K. J. Shea, J. Org. Chem., 1996, 61, 7438; (b) P. W. M. Sgarbi and D. L. J. Clive, Chem. Commun., 1997, 2157; (c) S. M. Sheehan, G. Lalic, J. S. Chen and M. D. Shair, Angew. Chem., Int. Ed., 2000, 39, 2714; (d) J. Cossy, S. BouzBouz, M. Laghgar and B. Tabyaoui, Tetrahedron Lett., 2002, 43, 823.

6 For some recent examples of fragmentation based approaches to bicyclic bridgehead olefins related to CP molecules and taxoids, see: $(a)$ T. Yoshimitsu, M. Yanagiya and H. Nagaoka, Tetrahedron Lett., 1999, 40, 5215; (b) T. Yoshimitsu, S. Yanagisawa and H. Nagaoka, Org. Lett., 2000, 2, 3751; (c) J. T. Njardarson and J. L. Wood, Org. Lett., 2001, 3, 2431.

7 G. Mehta, P. Talukdar and N. Mohal, Tetrahedron Lett., 2001, 42, 7663.

8 G. Mehta and J. Chandrasekhar, Chem. Rev., 1999, 99, 1437.

9 All new compounds reported here were duly characterized on the basis of spectroscopic data and elemental analyses. Diastereomers 13/14; $\mathbf{2 0} / \mathbf{2 1} ; \mathbf{2 3} / \mathbf{2 4}$ and $\mathbf{2 7 / 2 8}$ were all separated through chromatography on a $\mathrm{SiO}_{2}$-gel column.

10 R. H. Grubbs and H. Chang, Tetrahedron, 1998, 54, 4413.

11 For a review on the application of RCM in synthesis, see: S. K. Armstrong, J. Chem. Soc., Perkin. Trans. 1, 1998, 371. 